\title{
Beta-lapachone Prodrug ARQ 761
}

National Cancer Institute

\section{Source}

National Cancer Institute. Beta-lapachone Prodrug ARQ 761. NCI Thesaurus. Code C99146.

A synthetic, soluble prodrug of beta-lapachone, a poorly soluble, ortho-naphthoquinone with potential antineoplastic and radiosensitizing activity. ARQ 761 is converted to betalapachone (b-lap) in vivo. When b-lap is activated by $\mathrm{NAD}(\mathrm{P}) \mathrm{H}$ :quinone oxidoreductase-1 (NQO1) this agent creates a futile oxidoreduction, generating highly reactive oxygen species (ROS) that results in DNA damage. The activation of b-lap also causes hyperactivation of poly (ADP-ribose) polymerase-1 (PARP-1), an enzyme that facilitates DNA repair, accompanied by rapid depletion of NAD+/ATP nucleotide levels. As a result, a caspase-independent and endoplasmic reticulum (ER) stress-induced mu-calpainmediated cell death occurs in NQO1-overexpressing tumor cells. In addition, b-lap induces expression of the checkpoints activator E2F transcription factor 1 (E2F1) and thereby activates the E2F1-mediated checkpoint pathway that directly triggers apoptosis. As ARQ 761 is soluble and requires less solvent, this formulation may cause less hemolytic anemia associated with administration of the synthetic b-lap ARQ 501. 